This item was submitted to Loughborough's Research Repository by the author.

Items in Figshare are protected by copyright, with all rights reserved, unless otherwise indicated.

\title{
I know this one, but the answer is complex...
}

PLEASE CITE THE PUBLISHED VERSION

http://www.drs2016.org/239/

\section{PUBLISHER}

Design Research Society

\section{VERSION}

AM (Accepted Manuscript)

\section{PUBLISHER STATEMENT}

This work is made available according to the conditions of the Creative Commons Attribution-NonCommercialShareAlike 4.0 International (CC BY-NC-SA 4.0) licence. Full details of this licence are available at: http://creativecommons.org/licenses/by-nc-sa/4.0/

\section{LICENCE}

CC BY-NC-SA 4.0

\section{REPOSITORY RECORD}

Downs, Simon T.. 2019. "I Know This One, but the Answer Is Complex...". figshare. https://hdl.handle.net/2134/23531. 


\title{
Future-Focused Thinking
}

\section{I know this one, but the answer is complex...}

\author{
Simon Downs \\ ${ }^{a}$ Loughborough University School of Arts, English and Drama. \\ *s.t.downs@lboro.ac.uk
}

\begin{abstract}
There is a seductive logic in Abelson's 1979 paper Differences between belief and knowledge systems. This seduction comes in the form of an implicit promise that the formulation of a working epistemological address to the problems raised in the paper will give us a toolset that will blow away the fog of belief from the human landscape. This paper proposes that the very dynamism that makes the human landscape a swamp of wicked problems makes Abelson's conjecture however true they may be on a meta scale - an unproductive addition to the designers toolbox. It proposes that middle-range theories offer productive addresses to the complex systems and wicked problems that define our world.
\end{abstract}

Keywords: Design, Epistemology, Complexity, Systems. 


\section{Introduction}

This paper will address the topic of why a design epistemology is a beautiful and desirable thing, but in the case of communication design (graphics, illustration, typography, $\mathrm{HCl} / \mathrm{UX}$, etc.) the processes of cultural formation render it less useful than might be hoped for. Rendering any effective communications design epistemology as a small and local thing. A thing that needs to be refreshed through active processes of research with those people who use our products.

In his 1979 paper Abelson is looking for some species of absolute truth, an epistemological value shared across all groupings: his consensuallity (Abelson, 1979, p.356-7). While he makes a clear case for his logic in terms of the development of artificial intelligence this meta-truth has less utility than might be hoped for in the case of designers who need to access a contingent truth. That is to say the truth of the user: the culturally mediated truth of the those whose worlds we are engaging with.

That designers adopt this responsive stance is not a simple matter of politeness, some gentlemanly code of good manners for the designer to adopt so as not to upset the user. Rather, it is is a necessary function for effective transmission and reception of meaning. This stance is an emergent function of Second Order information flows (e.g. Second Order Cybernetics. Glanville, Von Foerster, et. al. ), which is to say the observer interferes with the observed by their mere presence. Designers must know their users to make this a fruitful change, while understanding that a successful change now changes the conditions for any future intervention. Design interventions handled without a cognisance of the user's lives will at best at best produce minor changes of behaviour, while at worst be met with incomprehension. Which connects with the simple truth that people are bound, at a very real biological and perceptual level, by the universe created from their cultural conditioning (Dennett's Homoncular Functionism, Dennett, 2014, p.93-95). Accepting this position creates a logic where the 'truth' of one culture may be completely 'false' for another, or more dangerously, completely unknowable. 
This will start to sound like a post-modern apologia for the position where all truth is equally valid: but it is not. This paper proposes that the Wicked Problems observed by Rittel are a very real symptom of culture composed of sets of interwoven, dynamic and self-organising human choices true within their own worlds but false in others: which is to say a Complex Adaptive System (CAS) showing Emergent Behaviour. Taking this position allows for the observed vitality and novelty of the cultures we inhabit, while at the same time explaining that for the inhabitants this culture it exhibits Epistemological (capital 'E') features of truth. ${ }^{1}$ Far from being beyond the epistemological pale, such systems are already proposed in social systems and their interactions: Goldman's W-knowledge or Mansell and Silverstone's Middle Range Theories to name two. This paper is not speaking against epistemology, but is promoting an epistemology that is fit for purpose as a design tool (Downs, 1, p.2009).

in talking about a fit for purpose design tool we're really talking about, at least from the viewpoint of a communication designer, is the devising of a systematic approach to validating truth about heterogeneous meaning for diverse cultures, for groups of people outside the design team (a basic problem in communication, what Abelson calls Existence beliefs). So while Abelson is (to be unkind) engaging in positivist modernist games in looking for a godlike perch from which to observe the fall of the sparrow, and the fine arts wish to occupy a realm of chaotic flux where there are no meta-narratives, this paper argues that for reasons stemming from the genesis of culture (in Complex Adaptive Systems) that Abelson's holistic epistemology, while not impossible, is not in itself a useful enterprise for designers. By contrast a middle range epistemology while 'not totally satisfying' (Abelson, 1979, p.362) is actually pretty useful in finding solutions that are meaningful for the people we co-design with.

\section{Not true, but useful.}

The author of this paper works in an art school of a quite traditional British model (which is to say more William Morris than Bauhaus or Vhkutmas). Arts theorists are systemically dominant in setting the philosophical agendas that practitioners research and teach from, as such the use of the word 'Truth' is something of an anathema.

The game at play, in such institutions, is the post-modern ironic one where truth is absent, meaning is negotiated and there are no meta-narratives. Which if one were to take at face value would leave graphic and communication design in a parlous state (the author has the work of David Carson in mind, but that may be unkind). Designers inhabiting a world where communication, if it were to happen, happens only erratically and not entirely in the way it was designed to do.

\footnotetext{
${ }^{1}$ Unless otherwise stated the author is treating knowledge as being those of the pre-Gettier values of Justified True Belief. Post-Gettier epistemological conversations on the subject are unresolved and the proper domain of philosophers not designers. See Mansell \& Silverstone, Merton, Goldstein, etc. for a discussion on the value of Middle-Range Theories.
} 
Communication Design (to borrow Gui Bonsieppe's term covering Graphics, Illustrations, etc.) is the child of the modernist upwelling of the late 19th and early 20th century. Finding its voice in the intensely systemic work of people like Muller-Brockman, Tschichold and Rockwell, and operating in a quasi-scientific manner where particular arrangements of form or treatments of type where considered to be inherently optimal for factual reasons. It was a field that had truth on its side. As Krippendorff (2004: 43) puts it, these modernist values, '... brought forth a technology that was believed to develop autonomously and progress naturally towards a better world for all the people that contributed to it.'

The exposure of the field to other cultures, times and places eroded these values, and leaves a subject where practitioners are trapped between the Scylla of modernist dogmatism and the Charybdis churning post-modern flux. We are a field in urgent need of working truths.

Beyond such practical concerns as the design of an illustration or street sign this debate is, in philosophical terms, problematic. On the one hand we might call on Wittgenstein's ghost to supply a rejection of the notion of personal languages (Candlish and Wrisley, 2014) ${ }^{2}$ or on the other we might look to Dennett's Intentionality (Dennett, 1971, p.87). ${ }^{3}$ What can't be argued is that across a spectrum composed of more or less effective levels of communication, communication does happen. ${ }^{4}$ Post-modernists tacitly admit this state.

Žižek (2006, p.16-17) notes in a discussion of Lacan's notion of partial truth that even the design of a toilet is culturally constructed to meet a culture's worldview, '...none of these versions (of toilets) can be accounted for in purely utilitarian terms: a certain ideological pereception of how the subject should relate to the unpleasant excrement that comes from within our body is clearly discernible in it (the design).' If we find the acceptance of the idea of partial truths and local rightness, in the works of post-modernists like Lacan and Žižek, then that would seem to indicate that considering this position has some utility.

This leaves both the Abelson agenda; of a TRUTH, universally acknowledged; and the opposing post-modern position, of less use than might be hoped for designers at work in the field. Abelson 1979 carries an internal logic derived from within it's own domain of knowledge (the development of Al systems, from the ground up first working principles: a methodology that has been overtaken by evolutionary, heuristic learning systems), however the logic applied in the paper is not a very human logic. It is an exclusive logic that demands ways before means. Krippendorf writing in 1969, observed that it was not possible to predict a design and it's nature was retrodictive. He points out that in design problems it is not possible to predict the solutions; or even the full set of stages leading to the solution; but that in retrospect the design process was always a logical set of steps:

'The introspective operation (a communication, including a designed one) provides a symbolic projection backward, so to speak, from an ultimate goal or objective to alternative acts of communication available at the present. This operation has

\footnotetext{
${ }^{2}$ E.g.. A lack of shared symbols deprives the author of the ability to even frame the context of their own private language.

${ }^{3}$ E.g. Communication is possible because we hold internal models of the minds of external agents.

${ }^{4}$ It seems cheeky to make the point that argument is only possible because communications happen.
} 
therefore been named "retrodiction." Communication praxiologies might thus be said to retrodictively justify their objects,...' (Krippendorff, 1969, p.115)

Which suggests that a design epistemology is not in a position to be predictive, but that there must be some form of truth that provides the user a position from which to recognise success at the end. This un-epistemic lack of clarity at the beginning of a job is not an unexamined proposition. Rittel's Wicked Problem Theory suggests that the notion of social truths that are contingent (while be real and pressing to those inhabiting the world bounded by that truth), fluid and amenable to modification and intervention. The interplay of Complexity Theory and Wicked Problem Theory is addressed later in the paper.

For an epistemology of visual culture to offer a viable support for graphic communications work it must account for the playful innovation of functional elements, by users (including designers who, let us not forget, are still users), elements that other users can still regard as meaningful and true, (i.e. signs that are not rendered meaningless by personal playfulness) (see Kuhn, 2009). This paper proposes that emergent effects arising from the very complexity of the large scale systems of interwoven feedback loops and agent choice that is Culture. Which can, on one scale, offer the observed ceaselessly churning cauldron of creativity that is the cultural world, while on the other scale offer the framework of meaning that feels as personal and true as a loved one's smile. An epistemology dealing with communication design needs to address truth on the level of the user. ${ }^{5}$

\section{What is Complex and what is Emergence?}

In the proceeding paragraph the terms 'emergent' and 'complex' are used, and they are used as specific technical terms from the domain of mathematics (and latterly computing, physics, biology, planning, engineering, social sciences, etc.). Terms which, while emerging from a positivist paradigm, are specifically constructivist in effect.

The study of Complex Adaptive Systems and the Emergence phenomena it creates have grown from a common realisation in the later part of the 20th century that the whole was not only often greater than the sum of its parts but often possesses an entirely different nature. A 'difference' that has the power to downwardly affect the individual parts that compose this novel system. For example the neurons in your head being effected by ideas they conjure for others or the people voting in an election being effected by the government they elect.

Consider the difference between systems that are merely complicated and those that are truly complex. An airliner is complicated, the Boeing 747 has something like 6,000,000 parts (Boeing.com, 2015) but the interactions of those parts is known and predictable. They are

\footnotetext{
${ }^{5}$ It has been suggested that there is a difference between technical and social problems in Visual Communication design, a suggestion which, while having a good deal of logic behind it, is countered by the work of Idhe (Multi-stable Design) and Latour (Instrumentality). Idhe and Latour propose that technologies find social relevance, often against their original design intent, which in turn lead to development cycles that are downwardly casual on social systems playing host to the technology. An argument which is a form of address to the debate started by Elizabeth Eisenstein and The Printing Press as an Agent of Social Change, and another example of Complex Adaptive Systems and Emergent effects.
} 
components with known and invariant functions. As such, the function of the parts in the whole is well described, the effect of the parts on the system is deterministic: this is a complicated system: but not complex. When you start to add interactions with other external systems (e.g. weather), which are themselves the end result of complex systems, and humans agents (with differing motivations and agendas) the combination of the plane, the weather and the agents becomes a complex system. It shows the potential for novelty (add pilots and the plane takes off), add passengers and it produces profit which has a downwardly casual effect on the other components of the system, it goes places (changes its state) and it makes changes to the dynamic of the complex system it inhabits (e.g. creates wake turbulence and contrails). This is now a complex dynamic system.

Much the same can be said of the social world, and by extension the cultural (Cilliers, Maturana, Luhman, Keller, etc.), and by further extension the visual cultural. As visual communication designers we can see that a completely viable design solution, tested by time, with an impeccable pedigree can fail as society changes or it is applied in a new technical environment (Downs 2007 and 2009 for a discussion on emergent typographic changes). The conditions that supported the existing conditions of epistemological rightness have changed and invalidated a previously 'right' solution. Krixpendorf's retrodiction shows us the error of assuming a previously functional epistemological order is still correct.

Not that this is a particularly new interpretation of the problem. This question of order arising spontaniously from disorder without authorship is Adam Smith's Invisible Hand played out on the world, it is David Hume's Copy Principle simultaneously extended across heterogeneous populations. This is an old set of arguments that has recently, through mathematical models run on increasingly fast computers, found new relevance. (see, Goldstein 1999 for a history). The question might be simply rendered as: How do individuals keep up with the endless mutation in culture? How do they understand the changes that happen beyond their sight, so that when the change comes into view it is comprehensible and right? Complexity theory offers us a model of this change that is consistent with observed social phenomena (Latour 2007, Keller 1994) and with design practice.

\section{What is Complex and what is Emergence?}

In the proceeding paragraph the terms 'emergent' and 'complex' are used, and they are used as specific technical terms from the domain of mathematics (and latterly computing, physics, biology, planning, engineering, social sciences, etc.). Terms which, while emerging from a positivist paradigm, are specifically constructivist in effect.

The study of Complex Adaptive Systems and the Emergence phenomena it creates have grown from a common realisation in the later part of the 20th century that the whole was not only often greater than the sum of its parts but often possesses an entirely different nature. A 'difference' that has the power to downwardly affect the individual parts that compose this novel system. For example the neurons in your head being effected by ideas 
they conjure for others or the people voting in an election being effected by the government they elect.

Consider the difference between systems that are merely complicated and those that are truly complex. An airliner is complicated, the Boeing 747 has something like 6,000,000 parts (Boeing.com, 2015) but the interactions of those parts is known and predictable. They are components with known and invariant functions. As such, the function of the parts in the whole is well described, the effect of the parts on the system is deterministic: this is a complicated system: but not complex. When you start to add interactions with other external systems (e.g. weather), which are themselves the end result of complex systems, and humans agents (with differing motivations and agendas) the combination of the plane, the weather and the agents becomes a complex system. It shows the potential for novelty (add pilots and the plane takes off), add passengers and it produces profit which has a downwardly casual effect on the other components of the system, it goes places (changes its state) and it makes changes to the dynamic of the complex system it inhabits (e.g. creates wake turbulence and contrails). This is now a complex dynamic system.

Much the same can be said of the social world, and by extension the cultural (Cilliers, Maturana, Luhman, Keller, etc.), and by further extension the visual cultural. As visual communication designers we can see that a completely viable design solution, tested by time, with an impeccable pedigree can fail as society changes or it is applied in a new technical environment (Downs 2007 and 2009 for a discussion on emergent typographic changes). The conditions that supported the existing conditions of epistemological rightness have changed and invalidated a previously 'right' solution. Krixpendorf's retrodiction shows us the error of assuming a previously functional epistemological order is still correct.

Not that this is a particularly new interpretation of the problem. This question of order arising spontaniously from disorder without authorship is Adam Smith's Invisible Hand played out on the world, it is David Hume's Copy Principle simultaneously extended across heterogeneous populations. This is an old set of arguments that has recently, through mathematical models run on increasingly fast computers, found new relevance. (see, Goldstein 1999 for a history). The question might be simply rendered as: How do individuals keep up with the endless mutation in culture? How do they understand the changes that happen beyond their sight, so that when the change comes into view it is comprehensible and right? Complexity theory offers us a model of this change that is consistent with observed social phenomena (Latour 2007, Keller 1994) and with design practice. 


\section{Not right or wrong, but good enough ${ }^{6}$}

Much of what historically seemed like the inescapable logic of graphic design (epistemology played out in the world) is the logic of a past culture and production ecology. Where once there existed an inescapable bag of 'good design' rules that we could rely on to function for 'everyone' we find ourselves in the position of having to design in the dialect of a specific user group: not just the formal graphic language of that culture (e.g. Manga and Kawii in Japan) but the local one (is your Manga Shōnen or Yuri?). '...communication arises in the concurrent unfolding of communication constructions, simultaneously held by its participants', inter-twining communication practices.' (Krippendorff, 1994, p.85)

The earlier logic of design truths has become heavily eroded by technically mediated social changes that have degraded the worth of simpler all embracing epistemologies (or 'functionalist design criteria' as Krippendorff would have it):

'These developments, obviously supported by current technology, now radically undermine functionalist design criteria, the validity of mechanistic/causal explanations of human behaviour and the role of experts in these traditional domains.'

(Krippendorff, 2004, p.44).

Successful communication happens through the existence of a common ground between both parties; in this case a designer and user; with a common set of 'dependencies'. (Wittgenstein via Candlish \& Wrisley (2014), Krippendorff (1994)). Dependencies are conditions that must be fulfilled if an operation is to proceed to its next stage. In complexity theory this framing of what might come next by what has come before is called 'Sensitivity to Initial Conditions' (see below). This sensitivity explains why a working design will have to acknowledge its own past; e.g. the chain of signification from which it arises, the design processes it uses, its institutional and contextual aptness; but is very unlikely to function in exactly the same way as its ancestor (because the ancestor is now part of the current design's initial conditions and therefor the dependancies are not the same). Perhaps failing where the ancestor became a standard of good design. The past becomes an index but not a proof.

This is acknowledged by Middle Range Theories (Mansell and Silverstone) and W-Knowledge where institutional information is counted as knowledge within a specific institution because it meets (locally), the epistemological conditions of knowledge, without achieving Abelson's global qualities. Designer's don't need a full view of total truth (though that would be useful) in order to function as designers, but they absolutely require a view of the dependencies of

\footnotetext{
${ }^{6}$ This process of designers searching for a working, as opposed to perfect, solution has a degree of equivalency to the notion of Satisficing (as used in fields like Economics and Information Sciences) which acknowledges that social agents will often compromise the desire for a perfect best of class solution that is either; distant, unachievable, or currently unknowable; for one that is merely good enough or fit for a task in hand. Simon $(1971$, p.71) has defined Satisficing as that process of decision making 'through which an individual decides when an alternative approach or solution is sufficient to meet the individuals' desired goals rather than pursue the perfect approach'. There is a much longer argument to be had as to whether Satisficing is compatible with Wicked Problem theory, as that theory explicitly points out that there are no optimum formulations to a problem, and strongly implies that all solutions are contingent on both the designer's worldview and the user's lived experience. See Ritchey, 2005/ revised 2013.
} 
the user group. When thinking of design we need something, 'close enough to observed data to be incorporated in propositions that permit empirical testing.' (Merton, 1968, p.39)

Reflecting on the value of W-knowledge in everyday life Goldman states: ${ }^{7}$

'A second and more important reason is that people's dominant epistemic goal, I think, is to obtain true belief, plain and simple. They want to be informed (have true belief) rather than misinformed or uninformed.... But the rationale for getting such evidence is to get true belief.' (Goldman, 2003, p.24)

We can see this in action in design jobs. The user and the designer, sharing some common cultural frameworks, will approach any task of coding or decoding a design requiring that common dependencies be met in order for communication to happen. So when Chan (2009) describes the work done in Australia, through public information campaigns intended to raise awareness of HIV/Aids, he notes that the graphic languages applied:

'tend to address the risk of infection in a manner which informs rather than invokes fear, shock or taboo.... This locates government funded HIV/AIDS intervention programmes in a morally charged arena open to public criticism and scrutiny on promiscuity, sexuality, and substance abuse.' (Chan, 2009, p.3586)

This scrutiny left the designers working for government funded initiatives working with a reduced set of viable semantic elements at their disposal, elements that failed to meet the target users dependencies for communication. Whereas other groups designing from within the Gay community had the freedom to design for the dependencies of the user.

'Graphics designed and produced by nongovernment organisations (NGO) or community-based organisations ( $\mathrm{CBO}$ ) for specific populations, e.g. gay men, are more likely to use images and language appropriate for the target group, and contrast sharply with generic images and messages used in national campaign materials for mainstream society produced by the health authorities.' (Chan, 2008, p.3586)

At the end of his study Chan states:

'Generic health messages which are translated from the dominant vocabulary more often than not fail to resonate with audiences from different cultural backgrounds. Similarly, the use of imagery requires considerable understanding and sensitivity towards the cultural experience and expectations of the community.' (Chan, 2009, p.3593)

Communication designers of all kinds are trained to understand the requirement for semantically meaningful content. Complexity and emergence give us a mechanism that explains how meaning happens; explaining not only the co-evolution of meaning between user and creative, but also the appearance of novel meaning is a persistent, normal, and yet, completely unpredictable feature of the system of graphic languages. It explains meaning without godlike authorship, widely spread across the whole group of participants: designer and users.

\footnotetext{
${ }^{7}$ Goldman's epistemological formulation has Strong (S) knowledge which is an epistemological absolute contrasted with Weak (W) knowledge, which is knowledge that is sufficient for the specific task at hand.
} 


\section{Complex and Emergent}

In the early days of the Twentieth Century it must have seemed that Bertrand Russell had good reasons to suppose that by knowing and naming the parts of a whole we could know the its nature.

'We may say that this is the characteristic merit of analysis as practised in science: it enables us to arrive at a structure such that the properties of the complex can be inferred from those of the parts.1 And it enables us to arrive at laws which are permanent, not merely temporary and approximate. This is an ideal, only partially verified as yet; but the degree of verification is abundantly sufficient to justify science in constructing the world out of minute units.' (Russell, 1992, p.285-286)

This quote has a special relevance because in the footnotes that accompany it Russell specifically (negatively) addresses the concept of Emergence.

'Dr C. D. Broad, in The Mind and its Place in Nature, lays stress upon what he calls "emergent" properties of complexes-i.e. such as cannot be inferred from the properties and relations of the parts. I believe that "emergent" properties represent merely scientific incompleteness, which would not exist in the ideal physics. It is difficult to advance any conclusive argument on either side as to the ultimate character of apparently "emergent" properties, but I think my view is supported by such examples as the explanation of chemistry in terms of physics by means of the Rutherford-Bohr theory of atomic structure.' (ibid)

Russell was wrong. Given a complex enough system, especially one that interacts with other systems, unexpected things will happen. ${ }^{8}$ Things that will make absolute sense if we retrospectively unpack the chain of events supporting their genesis, but which are not predictable. This property is called emergence.

There are several standard features of Complex Adaptive Systems. Chan (2001, p.3-6) identifies the following:

1.Distributed Control. There is no master plan inherent in a CAS. There is no leader or controller. The control comes from the overall behaviour of the sum of the agents in the system.

2.Connectivity. The elements in the system affect each other. The system which is the paper you are reading: thought, language, page, type, language, image functioning as a whole where the disparate elements don't.

3.Co-evolution. The actions of system elements affect change in the other system elements. These interactions affect the total system, causing changes in the future behaviour of system's agents. E.g. Users respond to a design, and this response causes an evolution in all future possible designs.

4.Sensitive Dependence on Initial Conditions. Small changes in the initial conditions of the system have large effects down the line: this is the butterfly effect of pop culture. This

\footnotetext{
${ }^{8}$ See The Law of Truly Large Numbers.
} 
sensitivity to initial states is one of the reasons why CAS' are essentially unpredictable. This sensitivity doesn't deny elements of determinism, but recognises that the determinisms may be negated or amplified by other parts of the system. So a fine piece of craft (image making or typography) may not make the appropriate connections with the intended user.

5.Emergent Order. Individual elements forge an order without central control: e.g. atoms forming molecules, forming proteins, forming organisms.

6.The phenomena of 'Far From Equilibrium'. Chan $(2001, \mathrm{p} 6)$ offers this definition of 'Far From Equilibrium':

'In 1989, Nicolis and Prigogine showed that when a physical or chemical system is pushed away from equilibrium, it could survive and thrive. If the system remains at equilibrium, it will die. The "far from equilibrium" phenomenon illustrates how systems that are forced to explore their space of possibilities will create different structures and new patterns of relationships.'

Complex Adaptive Systems have been suggested as the common model for a diverse range of systems defined by the interrelationship of multiple elements (Langton, Holland, Kauffmann, etc.). Langton notes, 'When you peel everything back they end up looking the same. You can literally map one model into another.' The diversity of medium hides the commonality of function.

Studies of communications as CAS' have generated the following features specific to communications operations: 're-inforcement learning, self-organisation, selection, coevolution through structural coupling, and level formation.' (Steels, 2000, p.1) Figure 1 shows a mapping of CASs to visual cultural operations and the technical means that support them. 


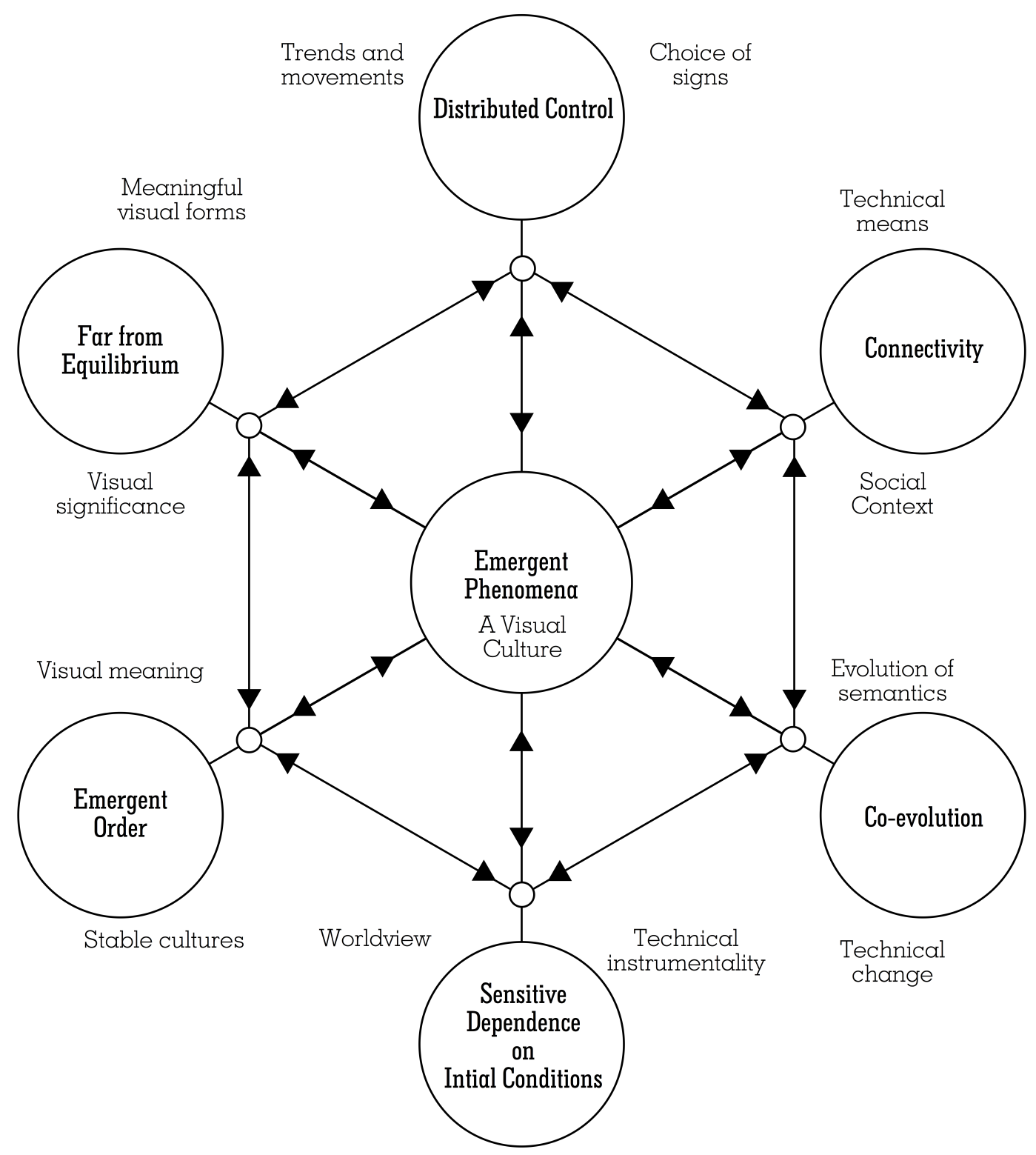

Figure 1:Figure 1 shows a mapping of CASs to visual cultural operations and the technical means that support them

The 'Emergent Order' mentioned above is a physical reality which when described seems unfeasible, too good to be true and some species of snake oil. Physist Doyne Farmer describing Emergence stated, 'It's not magic...but it feels like magic.' (Farmer, quoted in Waldrop, 1992, p.279). Our intrinsic life experience seems to tell us that order is hard to achieve, the result of labour and is unnatural. But when we consider that we experience our lives with evolved (not designed) sense organs, consider our lives with collections of neurons that have self-organised to become us, see weather patterns play across the globe without direction, in an atmosphere that shouldn't chemically exist (Prigogine's far from equilibrium), we can see that self-organised Emergent Order is very much part of our world (see, Waldrop, Maturana \& Varela, Langton, Holland, et. al.) 
There are many competing definitions of Emergence ranging from the brief, 'much coming from little' (Holland, 1998, p.17) to the wildly conflicting. For clarity I will be using Jeffrey Goldstein's definition: from the inaugural issue of the journal Emergence, as quoted in Corning (2002, p.7): partly because it is widely and positively cited, but mostly because it is both coherent and clear. Goldstein defines the following characteristics as Emergence:

'(1) radical novelty (features not previously observed in the system); (2) coherence or correlation (meaning integrated wholes that maintain themselves over some period of time); (3) A global or macro "level" (i.e., there is some property of "wholeness"); (4) it is the product of a dynamical process (it evolves); and (5) it is "ostensive" - it can be perceived.' (ibid)

Corning notes that, 'For good measure, Goldstein throws in supervenience downward causation'. (ibid)

For example in a communication design context:

1.The radical novelty is the difference in form and effect between the parts and the sum of those parts operating together. The configuration of the components forming El Lissitzky's Beat the Whites with the Red Wedge (Figure. 2) demonstrate radical novelty not observed by any other formulation of these elements.

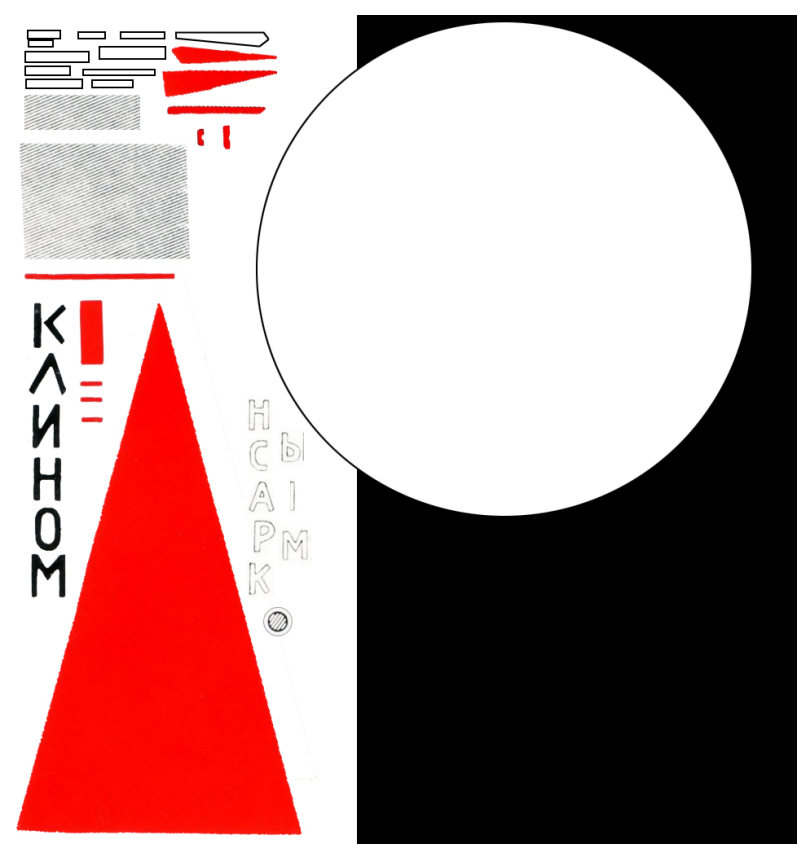

Figure 2: The individual elements that compose the Poster individually show none of the effective qualities that the composed whole does.

2.Once established these elements (and this formulation of the elements) is coherent (a discrete thing with a wholeness) with the ability to hold itself together over time. The coherence of Beat the Whites... is retained across the years.

3.We can see the macro level of 'wholeness' in this poster because we can identify it as itself, and not as a collection of elements, even though the elements are clearly present the 
whole is there too. It is itself and not something other. (Figure. 3 Even thought the context has changed the original poster is itself, and not another (even though the other refers to the original).)
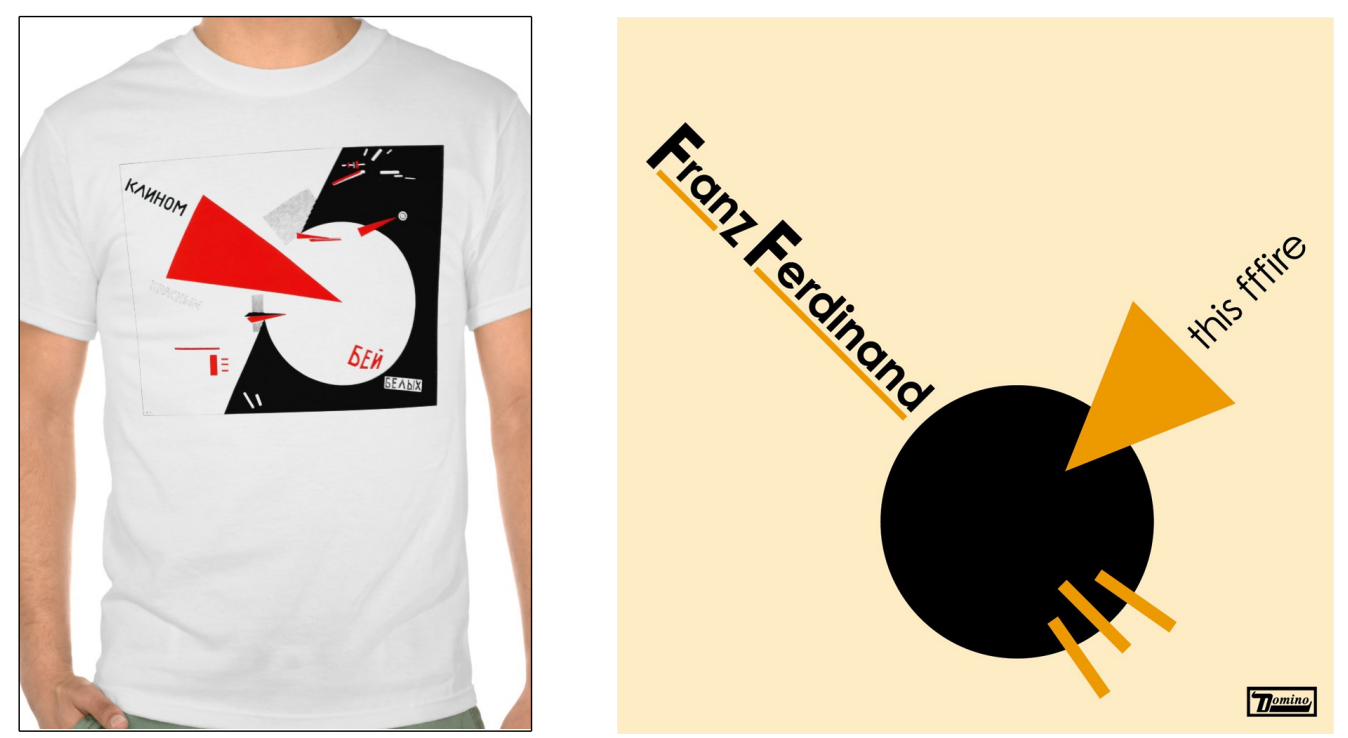

Figure 3: We can identify the original element as itself, and not as a collection of elements, even though the elements are clearly present the whole is there too. It is itself and not something other.

4.Beat the Whites... did not emerge from nothingness, it is a work connected to earlier works and from earlier influences (e.g. Chagall Fig.4), it's design and construction was a dynamic event, and it contributes to other works. 


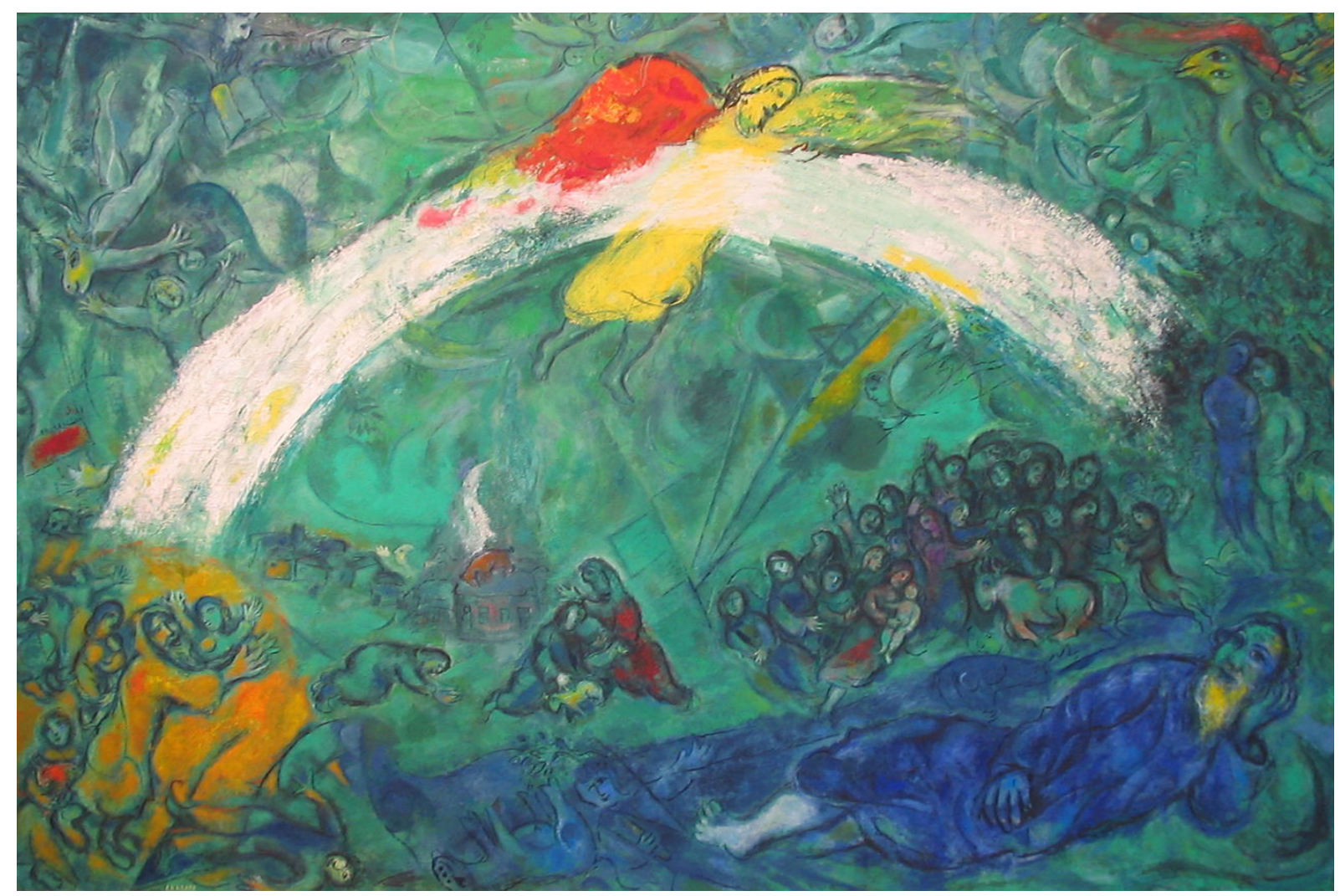

Figure 4: La Genèse et I'Exode. (Musée National, Cimiez, France.) By Marc Chagall. Showing similar composition techniques to those that influenced El Lissitzky. E.g. Dynamic elements playing across divided colour fields.

5.The poster is 'ostensive', we can perceive it as having an existence beyond that of the things around it.

6.The poster is also heavily influential in setting the dependencies for other iterations of poster design that follow it. It is downwardly casual on the environment that made it, and on the field that supports it.

It becomes clear that communication design, as both field and as product, shows the characteristics of emergence. This emergent order in communication design comes from the action of a Complex Adaptive System (culture) and the elements that compose it. As a response Rittel's Wicked Problem theory becomes a logical response to dealing with the contingencies imposed on possible design interventions by the nature of a cultural CAS (e.g. no single epistemological truth, creating hard to define problems, and floating truths in user's lives).

It is proposed in figure 5 that not only do Emergent systems arise from Complex Adaptive Systems and, in social and cultural systems at least, set the conditions for Wicked Problems, but that the resolution (or lack of resolution) of these problems sets the conditions for the next iteration of the CAS. In short we see a range of social and cultural feedback loops in which the communication design interventions modify the initial conditions of the system (a 
standard characteristic of CAS and Emergence in social systems). As Rittel and Webber (1973, p.159) put it:

'We have been learning to see social processes as the links tying open systems into large and interconnected networks of systems, such that outputs from one become inputs to others.'

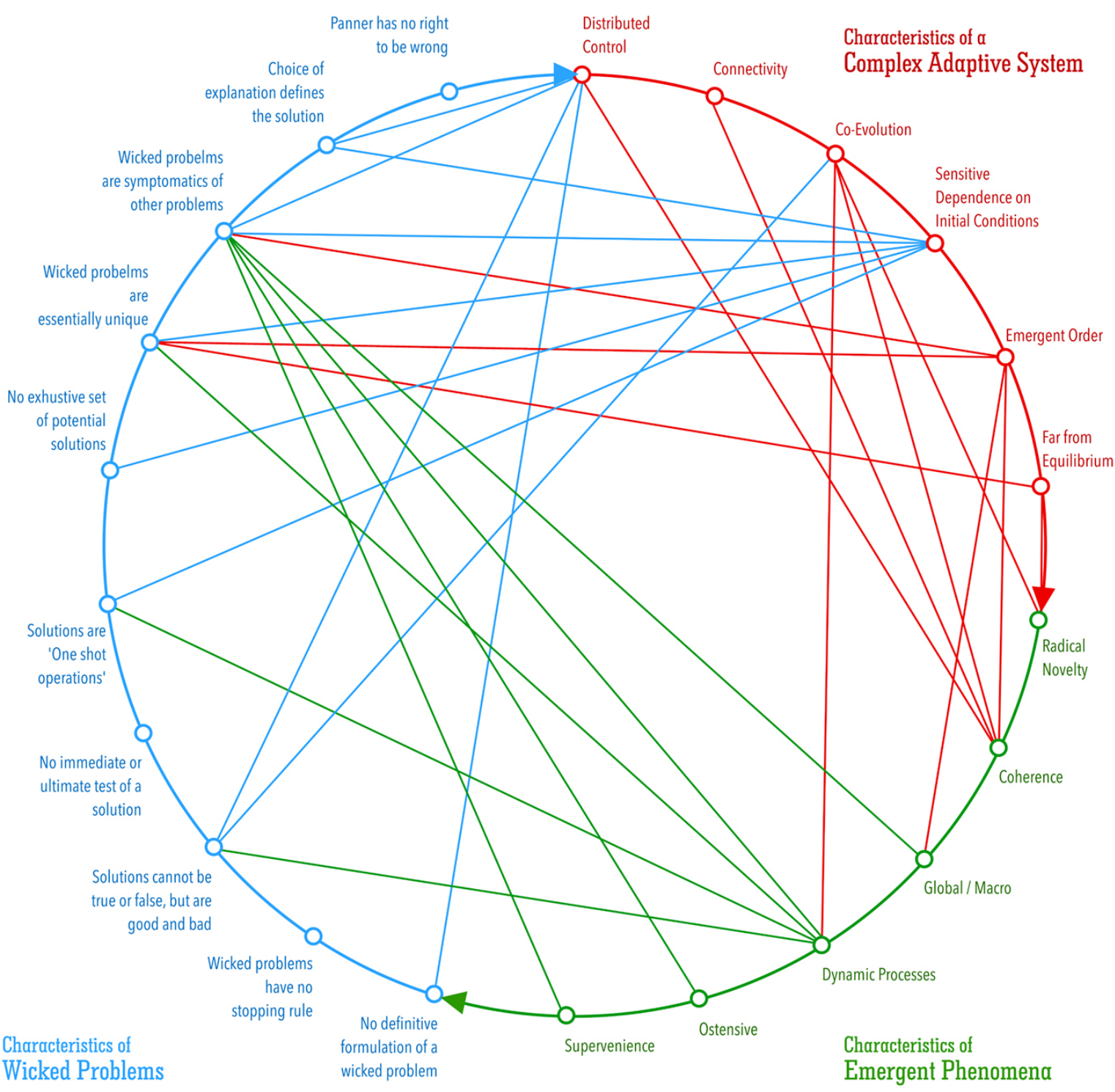

Figure 5: A diagram showing the cyclic and interconnected nature of the elements that form Complex Adaptive Systems, Emergent Properties, and Wicked Problems.

This leads us to see that the dependencies of communications emerges from common networks of meaning (semantics), that are formed by the action of complex adaptive systems. Designing for such systems needs to acknowledge the dependencies are the 
outputs of previous cultural activities, this can be done through treating communication design problems as wicked. Rittel defines wicked problems as:

'(1) Wicked problems have no definitive formulation, but every formulation of a wicked problem corresponds to the formulation of a solution.(2)Wicked problems have no stopping rules.(3) Solutions to wicked problems cannot be true or false, only good or bad.(4) In solving wicked problems there is no exhaustive list of admissible operations.(5) For every wicked problem there is always more than one possible explanation, with explanations depending on the Weltanschauung of the designer.(6) Every wicked problem is a symptom of another, "higher level," problem.(7) No formulation and solution of a wicked problem has a definitive test.(8) Solving a wicked problem is a "one shot" operation, with no room for trial and error. (9) Every wicked problem is unique.(10) The wicked problem solver has no right to be wrong-they are fully responsible for their actions.' (Rittel as cited in Buchanan, 1992, p.16)

Taking design epistemological truth to be culturally defined; regarding the cultural definitions of truth to be emergent from the operation of agents making up the system; then the observations that Rittel made become meaningful positions from which to assess local epistemological values. More than this they suggest that this painstaking approach of codesign is an example of the only class of solution from which we could establish meaningful epistemological value for communication design.

In conclusion, this paper suggest that a workable communication design epistemology is, by necessity a small and humble thing. Perhaps phrased as: 'A communication design solution is epistemologically valid if it works for the intended user.' A statement that is brief, recursively cute, but hides a thorny thicket of snares for the unwary or time pressed designer. There are no reasonable algorithmic or epistemic shortcuts. If our practice is to progress we need to build time for close engagement with those who will use our work.

Acknowledgements: the support of Dr. Claire Lerpiniere, has been invaluable in writing this paper.

\section{References}

Abelson, R. P., (1979) Differences between belief and knowledge systems. Cognitive science (Vol 3 Iss 4) pp. 355-366.

Boeing 747 promotional website, Boeing 747-8, http://www.boeing.com/commercial/747/ (Accessed 27th of October 2015).

Buchanan, R., (Spring, 1992) Wicked Problems in Design Thinking, Design Issues (Vol. 8, No. 2), pp. 521.

Candlish, S and Wrisley, G., "Private Language", The Stanford Encyclopedia of Philosophy (Fall 2014 Edition), Edward N. Zalta (ed.) http://plato.stanford.edu/archives/fall2014/entries/privatelanguage/, (Accessed 27th of October 2015).

Chan, L. And Donovan, R., (2009) Designing the Other: Australian HIV/AIDS Campaigns and Asian Gay Men. The Proceedings of IASDR 2009, Design / Rigour \& Relevance. Osaka University. pp. 3585 3594.

Chan, S., (2001) Complex adaptive systems. ESD. 83 Research Seminar in Engineering Systems pp. 1-9. 
Corning. P A., (2002) The Re-emergence of "Emergence": A Venerable Concept in Search of a Theory. Complexity (Vol 7, Iss 6) pp. 18-30.

Dennett, D., (Feb. 25, 1971) Intentional Systems. The Journal of Philosophy, Vol. 68, No. 4. pp. 87106.

Dennet, D., (2013) Intuition Pumps and Other Tools for Thinking. Penguin Books.

Downs, S., (2007) Bikes Don't Break Legs-VisCom as an Emergent Phenomenon. Design and semantics of form and movement. DeSForM (2007) pp.56-61.

Downs, S., (2009) The Truth? - Working towards an epistemology of Visual Communications Design. The Proceedings of IASDR 2009, Design / Rigour \& Relevance. Osaka University. p. 3339-3350.

Holland, J H., (1998) Emergence: From Chaos to Order, Addison-Wesley Helix.

Goldman, A I., (2003) Knowledge in a Social World, Oxford University Press, Oxford.

Goldstein, J., (1991) Emergence as a Construct: History and Issues, Emergence (Vol 1, Iss 1), pp. 4972.

Keller, R. (1994) On language change: The invisible hand in language. Psychology Press.

Krippendorff, K., (1969) Values, Modes and Domains of Inquiry into Communication, The Journal of Communication, (Vol 19) pp. 105-133.

Krippendorff, K., (1994) A Recursive Theory of Communication, in: D. Crowley and D. Mitchell (Eds.), Communication Theory Today, Polity Press, Cambridge, UK. pp. 78-104.

Krippendorff, K., (2004) Intrinsic motivation and human-centred design. Theor. Issues in Ergon. Sci., (vol. 5, no. 1) pp.43-72.

Kuhn, W. (2009) Semantic engineering. Research trends in geographic information science. Springer Berlin Heidelberg. pp. 63-76.

Latour., B., (2005) Reassembling the Social - An Introduction to Actor-Network-Theory (2007 edition). Oxford University Press.

Merton, R K., (1968) Social theory and social structure. Simon and Schuster.

Ritchey, T., (2005, revised 2013) Wicked Problems - Modelling Social Messes with Morphological Analysis. Acta Morphologica Generalis (Vol 2 No.1),

https://www.researchgate.net/profile/Tom_Ritchey/publication/236885171_Wicked_Problems_ Modelling_Social_Messes_with_Morphological_Analysis/links/00463519e5f08e672a000000.pdf (Accessed 10 March, 2016)

Rittel, H., and Webber, M., (1973) Dilemmas in a General Theory of Planning, Policy Sciences (Vol 4). Russell. Betrand, (1927) Analysis of Matter (1992 edition), Routledge, London.

Steels., L., (2001) Language as a complex adaptive system, in (Volume Editor(s)): Schoenauer \& al, Lecture Notes in Computer Science. Parallel Problem Solving from Nature - PPSN-VI. SpringerVerlag, Berlin. Pp. 17-22.

Waldrop, M. M., (2006) Complexity - Science at the Edge of Order and Chaos, Simon \& Schuster.

Z̆̌zek, Slavoi., (2006) How to read Lacan, Granta Books.

About the Author:

Simon Downs trained as an illustrator of a particularly traditional school. Retraining through working as a digital animator, screen 
designer, digital illustrator and editorial designer. He became an academic and educator in 2001. He now researches the ways in which the social and technical co-produce viable working solutions. $\mathrm{He}$ is the Lead Editor of The Poster (Intellect Books), an Editor of TRACEY (Loughborough University) and a Director of the Drawing Research Network. He is also a trades union activist with U.C.U. 